ARTICLE

\title{
Imaging how thermal capillary waves and anisotropic interfacial stiffness shape nanoparticle supracrystals
}

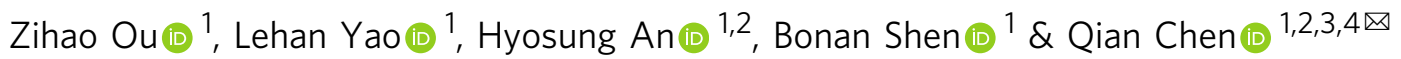

Development of the surface morphology and shape of crystalline nanostructures governs the functionality of various materials, ranging from phonon transport to biocompatibility. However, the kinetic pathways, following which such development occurs, have been largely unexplored due to the lack of real-space imaging at single particle resolution. Here, we use colloidal nanoparticles assembling into supracrystals as a model system, and pinpoint the key role of surface fluctuation in shaping supracrystals. Utilizing liquid-phase transmission electron microscopy, we map the spatiotemporal surface profiles of supracrystals, which follow a capillary wave theory. Based on this theory, we measure otherwise elusive interfacial properties such as interfacial stiffness and mobility, the former of which demonstrates a remarkable dependence on the exposed facet of the supracrystal. The facet of lower surface energy is favored, consistent with the Wulff construction rule. Our imaging-analysis framework can be applicable to other phenomena, such as electrodeposition, nucleation, and membrane deformation.

\footnotetext{
${ }^{1}$ Department of Materials Science and Engineering, University of Illinois, Urbana, IL 61801, USA. ${ }^{2}$ Materials Research Laboratory, University of Illinois, Urbana, IL 61801, USA. ${ }^{3}$ Department of Chemistry, University of Illinois, Urbana, IL 61801, USA. ${ }^{4}$ Beckman Institute for Advanced Science and Technology, University of Illinois, Urbana, IL 61801, USA. ${ }^{凶}$ email: qchen20@illinois.edu
} 
$\mathrm{T}$ he shape and surface morphology of crystalline nanomaterials are of central importance to determine their properties and applications $s^{1-4}$. For example, surface roughness alone has been shown to restrict phonon transport below the Casimir limit in crystalline Si nanowires ${ }^{3}$, and to dictate cellular adhesion and proliferation on zirconia nanofilms ${ }^{4}$. When it comes to "supracrystals" assembled non-covalently from nanoparticles (NPs, instead of atoms), the crystal shape and exposed external facets can play a key role in governing their architecturedependent properties ${ }^{1,2,5}$. For example, supracrystals composed of gold NPs have been shown to exhibit photon-plasmon coupling for potential applications in lasing and quantum electrodynamics $^{2}$, with their photonic modes encoded simply by the supracrystal shape. Numerous theoretical efforts have thus been focused on predicting the shape and surface morphology of crystals (i.e., crystal habit) developed during the growth process over the last few decades ${ }^{6,7}$. Regarding crystal shape, the Wulff construction rule is a prominent principle predicting the thermodynamically stable shape of a crystal by considering the collective effects of the surface energies of different facets of a crystal $^{7,8}$. Extensions of the Wulff construction rule to Winterbottom and Summertop constructions further predict the crystal shapes when the crystal growth is confined by substrates? Regarding surface morphology, a diversity of models on kinetic growth mode such as the Stranski-Krastanov, Frank-van der Merwe, and Volmer-Weber models weigh the balance of factors such as energy landscape of the crystal surface and surface diffusional dynamics, to predict whether building units attach layerby-layer (relatively smooth) or as islands (relatively rough) to growing crystals ${ }^{10,11}$.

However, despite the broad interest and extensive theoretical models in crystal habit, achieving a high level of control over it at the nanoscale requires addressing a few fundamental gaps. For supracrystals, controlling their surface roughness relies on understanding how NPs attach one by one onto the surface of a growing supracrystal during the self-assembly process in solution, which has been experimentally inaccessible due to the challenge to resolve the motions of individual NPs in solutions at the nanometer resolution ${ }^{12}$. As a result, supracrystal growth dynamics have been largely indirectly inferred from either ex-situ real-space measurements based on electron microscopy images of discrete stages of the growth process in dried samples ${ }^{13,14}$, or from in-situ reciprocal space characterizations like small-angle Xray scattering ${ }^{15}$. As to controlling supracrystal shapes, the encountered challenges are threefold. All the construction rules (e.g., Wulff, Winterbottom, Summertop) based on the thermodynamic stability require precise surface energy values of different supracrystalline facets as the inputs, which have not been experimentally measured ${ }^{8,9}$. Instead, these values were often estimated by a broken bond model assuming all the interparticle interactions are local, which could be hard to use due to the longrange nature of most colloidal interactions at the nanoscale ${ }^{16}$ or when the building units are highly anisotropic in shape (e.g., rods, prisms, and arrows) and render the interparticle interactions directional. Moreover, NPs can experience high diffusion barriers compared to atoms that these theories were originally established for, so that they might fall into kinetically trapped states which deviate from thermodynamic predictions ${ }^{17}$. Thirdly, simulations of their growth behaviors need to consider the nanoscopic morphology and interaction profiles of the NPs, and the mass and energy transport contributions-surface and volume diffusion, defect annealing, strain relaxation-all at once, which remains challenging and computationally expensive, especially in the absence of experimental data.

Here we utilize a recently developed technique of liquid-phase transmission electron microscopy (TEM) to experimentally map the kinetic and thermodynamic parameters key to crystal growth at the nanoscale, including the kinetic growth profile, facetdependent interfacial stiffness (the sum of surface energy and its second derivative), surface roughness, and interfacial mobility. Mapping of these otherwise inaccessible parameters is made possible by combining a capillary wave theory (CWT) and the capability to directly image a fluctuating surface of a growing supracrystal at the nanoscale using liquid-phase TEM. Liquidphase TEM can seal liquid samples against the high vacuum of TEM and capture the dynamics of nanosized entities at the nanometer resolution ${ }^{18,19}$. In this work, we use low-dose liquidphase TEM imaging to study the shaping process of NP supracrystals during post-nucleation growth. Aided by single-particle tracking, we find that the spatiotemporal fluctuations of the surface profiles of a growing supracrystal match quantitatively with CWT (Supplementary Movie 1), which has been prevalently used for equilibrated interfaces in micron-sized colloid ${ }^{20,21}$ and atomic systems ${ }^{22-26}$. This match is demonstrated here on the single NP level-only accessible with liquid-phase TEM-showcasing the capability of our method to minimize long-standing complications from beam or substrate. From the liquid-phase TEM movies, we capture an intriguing shift in the exposed facets (from (340) to (210)) during the supracrystal growth. Following CWT, we are able to measure the corresponding interfacial stiffness, which pinpoints the energetic driving force for this shift towards lower surface energy facets, leading to a smoother faceted morphology of the supracrystal. This real-space imaging and fluctuation analysis of a growing NP supracrystal provides guidance on encoding the shape and surface morphology of nanostructured materials from the building units and growth kinetics. Our workflow can be potentially extended to other phenomena governed by nanoscale interfacial fluctuations, from substrate guided crystal growth ${ }^{17}$, dendrite formation ${ }^{27}$, fusion of lipid vehicles ${ }^{28}$, to cellular compartmentalization ${ }^{29}$.

\section{Results}

Fluctuating surface of a NP supracrystal. In equilibrium, the free surface between two phases is determined by a balance of the flattening effect of surface energy and statistical fluctuations of local interface locations induced by thermal motions. In the framework of CWT, thermally induced capillary waves of different wavelengths superimpose into rough interfacial profiles, where equilibrium roughness is a tradeoff between the surface energy and thermal fluctuation 22,30 . In this context, the threedimensional (3D) supracrystal system whose nucleation pathway we characterized earlier ${ }^{31}$ under liquid-phase TEM serves as the model system to study nanoscale interfacial fluctuation (Fig. 1, Supplementary Note 1). The surface layer of the supracrystal interfaces with an aqueous suspension of dispersed NPs from which the supracrystal grows. The NP building blocks are gold triangular nanoprisms $(100 \pm 9.5 \mathrm{~nm}$ in side length, $7.5 \mathrm{~nm}$ in thickness, Supplementary Fig. 1). They are coated by negatively charged thiols and stay dispersed in deionized water due to an interprism electrostatic repulsion. An ionic strength increase to $\sim 0.5 \mathrm{M}$ screens the interprism electrostatic repulsion to induce the face-to-face stacking of the prisms into cylindrical columns standing vertical to the $\mathrm{SiN}_{x}$ substrate $^{31}$. As shown in our pairwise interprism interaction calculation (Supplementary Fig. 2), the column adopts the most stable configuration where all of the constituent prisms share the same $(x, y)$ centroid coordinates as the column. At this configuration, the net attraction energy is huge, of a magnitude larger than $20 k_{\mathrm{B}} T$. The energy penalty for the two adjacent prisms to deviate from this common centroid position by as small as $10 \mathrm{~nm}(\sim 10 \%$ of the prism side length) is already $2 k_{\mathrm{B}} T$, not favored when agitated only by thermal 


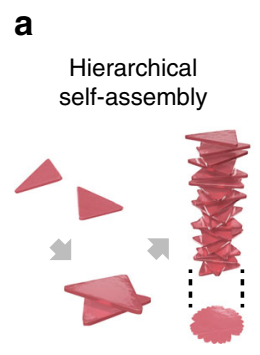

b

f

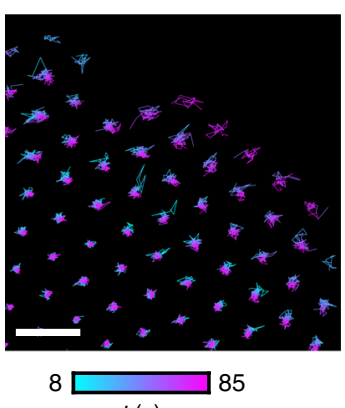

$t(\mathrm{~s})$

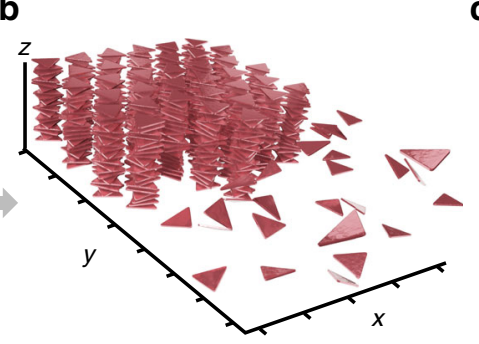

g
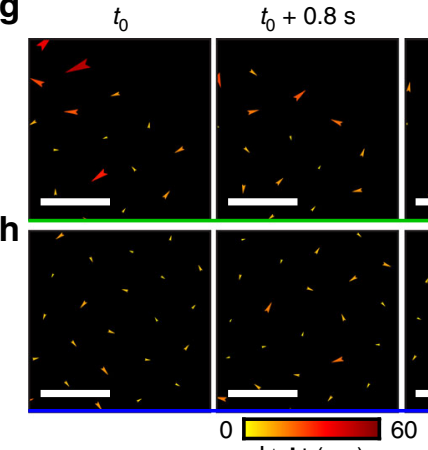

$\left|\Delta \mathbf{d}_{j}\right|(\mathrm{nm})$
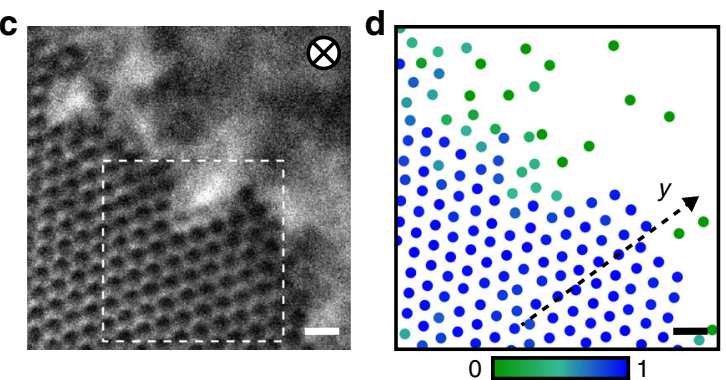

$\left|\psi_{6 j}\right|$

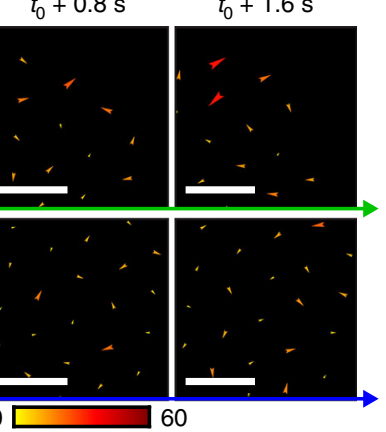

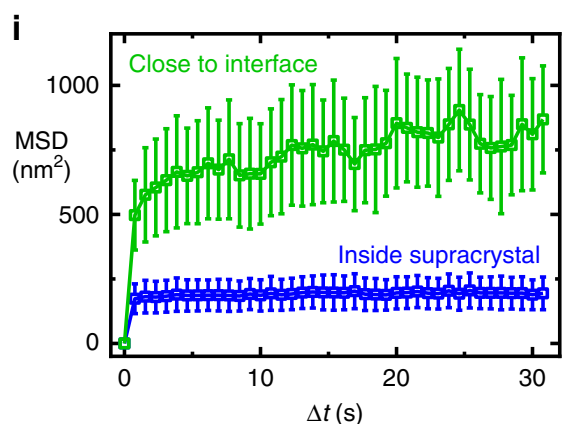

$\Delta t(\mathrm{~s})$

Fig. 1 A supracrystal-suspension interface visualized in real-time by liquid-phase transmission electron microscopy (TEM). a Schematic of the triangular prisms with favored face-to-face attraction stacking into columns in a misaligned fashion, leading to a circular projection in TEM. b Schematic of the supracrystal-suspension interface where the columns assemble laterally into an ordered 3D hexagonal supracrystal. c Liquid-phase TEM image of the supracrystal surface interfaced with the suspension of dispersed building blocks. $\mathbf{d}$ Plot (corresponding to the TEM image in $\mathbf{c}$ ) with single column positions tracked as filled circles, colored according to the jth column's local bond-orientational order parameter $\left(\left|\psi_{6 j}\right|\right)$. e Plot of $\left|\psi_{6 j}\right|$ across the $y$ direction labeled in (d). Red dashed line is drawn to guide the eye. $\mathbf{f}$ A trajectory map of columns corresponding to the boxed region in (c) measured from time-lapse TEM movies with color coded to elapsed time. $\mathbf{g}$, $\mathbf{h}$ Successive displacement maps of the columns close to the supracrystal-suspension interface ( $\mathbf{g}$ ) and inside supracrystal (h), where the length and color of the arrows denote $\left|\Delta \mathbf{d}_{j}\right|$, the magnitude of the displacement. i Plot of mean squared displacement (MSD) vs. time $\Delta t$ of the columns close to the supracrystal-suspension interface (green, mean \pm s.e.m. from 18 columns) and inside the supracrystal (blue, mean $\pm \mathrm{s}$. e.m. from 12 columns). Scale bars: $200 \mathrm{~nm}$.

fluctuations (Supplementary Fig. 2d, Supplementary Note 2). Accordingly, each column can be treated as one cylindrical object fully described by its projected $(x, y)$ centroid coordinates in our interfacial fluctuation analysis. Variations of the cross-sectional shape of the column do not affect the analysis either because the intercolumn interactions are radially symmetric (Supplementary Note 2). The number of constituent prisms in the columns is estimated to be $20-30$, resulting in a circularly shaped projection of the columns due to sufficient orientational randomness of the prisms $^{31}$. The columns further order into a large-scale hexagonal lattice, whose boundary is vertical to the view, so that we can track their fluctuation in the $x y$ plane at the single particle level over time (Fig. 1a-c, Supplementary Figs. 3-4, Supplementary Note 1-3, Supplementary Movie 2). We use low-dose liquidphase TEM protocols that our group has developed for NP crystallization studies (dose rates of $3.7-14.8 \mathrm{e}^{-} \AA^{-2} \mathrm{~s}^{-1}$ ) in a closely-sealed $\mathrm{SiN}_{x}$ liquid chamber ${ }^{31,32}$. These imaging conditions have been shown to keep the thiols and NPs intact upon electron beam illumination ${ }^{31,32}$. The supracrystal spans several microns in the $x y$ plane (Supplementary Fig. 5) and has a clear global six-fold rotational symmetry as suggested by the fast Fourier transform (FFT, inset in Supplementary Fig. 5a).

From the real-space TEM movies, we track the positions of a total of $\sim 220$ columns per frame with nanometer resolution (Fig. 1c, d and Supplementary Fig. 6, Supplementary Note 4), from which we confirm the dramatic changes of local structural order and motions of columns across the interface. Quantitatively, the supracrystal region exhibits a high and uniform local structural order as characterized by a six-fold bond-orientational order parameter ${ }^{33}$. This parameter $\psi_{6 j}$ is calculated as $\psi_{6 j}=$ $\frac{1}{Z_{j}} \sum_{k=1}^{Z_{j}} e^{i 6 \beta_{j k}}$ for the $j$ th column, in which $Z_{j}$ is the number of nearest neighbors and $\beta_{j k}$ is the angle of the bond linking the $j$ th column and its $k$ th neighbor. The magnitude of $\psi_{6 j}$ (i.e., $\left.\left|\psi_{6 j}\right|\right)$ measures the bond-orientational order on the single column level. Based on this analysis, $92 \%$ of the columns inside the supracrystal have $\left|\psi_{6 j}\right|>0.8$ (Supplementary Fig. 5a-c and Supplementary Note 5). The value of $\left|\psi_{6 j}\right|$ decreases sharply to zero within $\sim 300$ $\mathrm{nm}$ along the $y$ direction (labeled in Fig. 1d) from the supracrystal center to its boundary (Fig. 1e and Supplementary Fig. 5d-f, Supplementary Note 5). As shown in Fig. If, the columns close to the supracrystal surface are surrounded by fewer nearest neighbors and exhibit more translational freedom than those in the center of the supracrystal (Fig. 1g, h and Supplementary Fig. 7, Supplementary Note 6, Supplementary Movie 3). For more clarity, we plot the mean-squared displacements (MSD) versus time $(\Delta t)$ of the columns (Fig. 1i). For the columns in the supracrystal, the MSD reaches a plateau of $200 \mathrm{~nm}^{2}$ due to local confinement to the lattice sites, while for the columns near the surface, the MSD increases first and then flattens at $\sim 750 \mathrm{~nm}^{2}$, confirming more translational motions due to the less coordinated environment.

Mapping spatiotemporal fluctuations of surfaces. At each frame, we delineate the supracrystal surface by determining which columns are connected to each other and evaluating the local order of connected columns. Here the instantaneous profiles of 
a

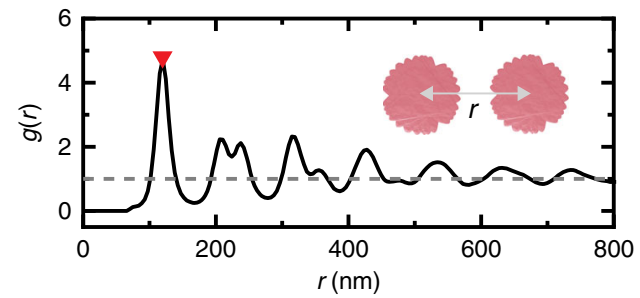

b

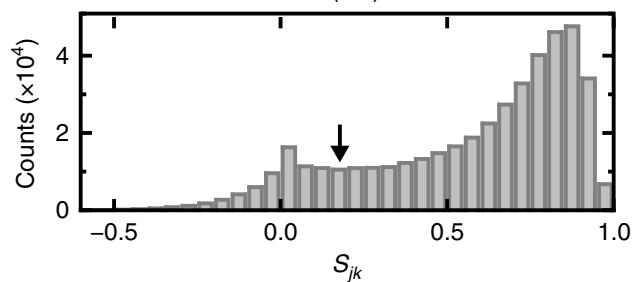

C

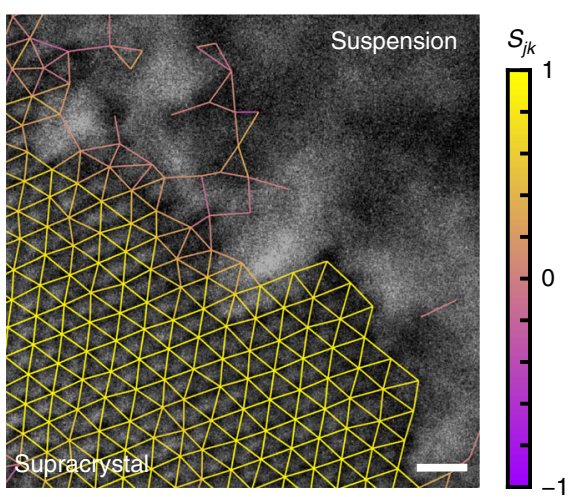

d
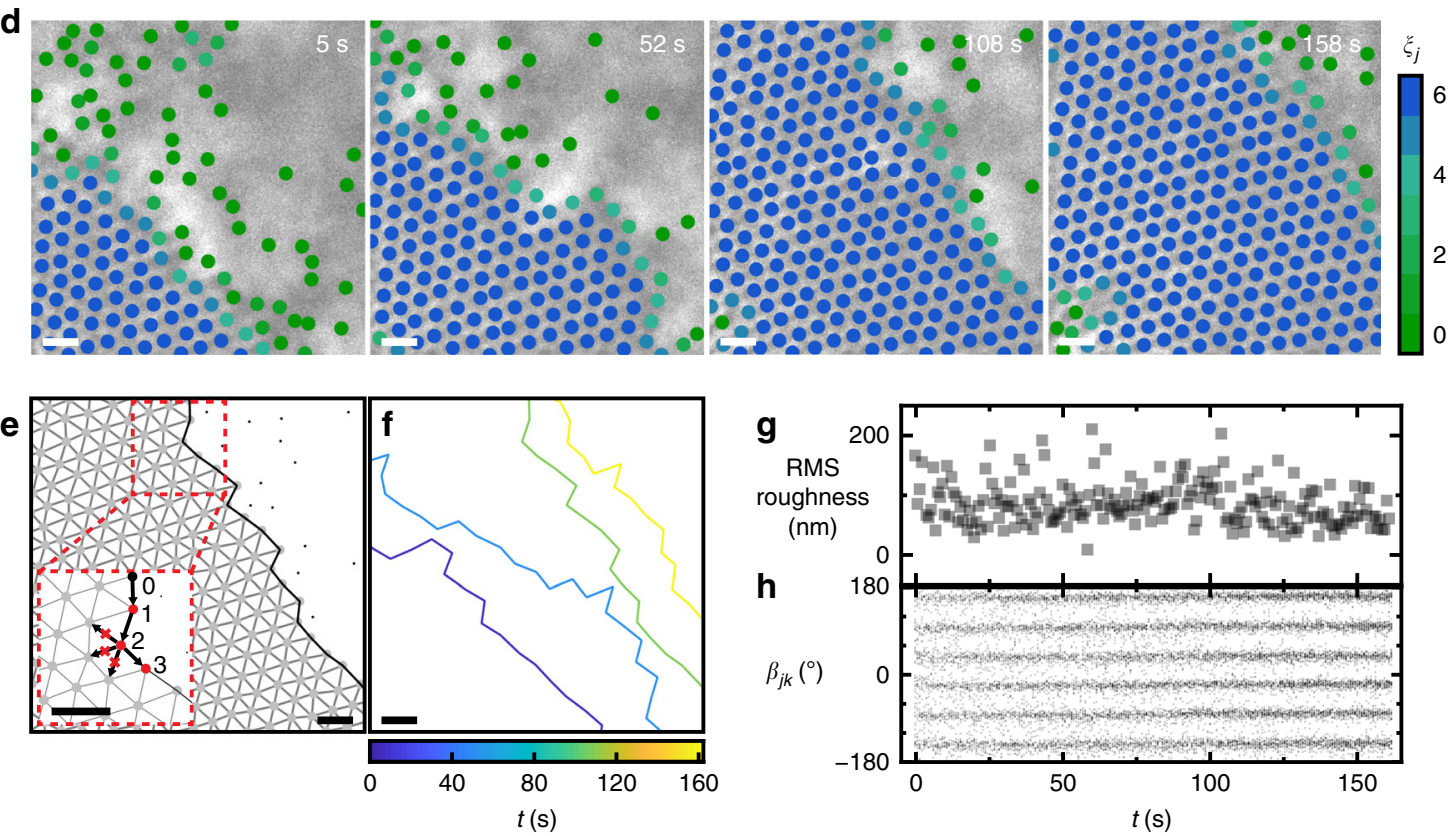

Fig. 2 Profiling of the surface layer in the supracrystal system. a Radial distribution function $g(r)$ as a function of $r$ (defined in the inset schematic) for the supracrystal computed from Supplementary Movie 2. The red triangle denotes the $r$ value corresponding to the lattice constant $\sigma$. $\mathbf{b}$ Histogram of the connectivity $S_{j k}$ computed from Supplementary Movie 2, with $S_{c}$ denoted with the black arrow. c Liquid-phase TEM image of the supracrystal interfaced with the suspension overlaid with the bond connection network color labeled according to $S_{j k}$ values. d Time-lapse TEM images overlaid with tracked column positions (filled circles colored to the values of $\xi_{j}$ for the $j$ th column). e The bond connection diagrams corresponding to one TEM image in $\mathbf{d}$ (108 s), where columns that belong to the supracrystal are labeled as gray filled circles and others are labeled as black dots. The supracrystal surface, i.e., the outermost surface layer of the supracrystal, is traced as the black line. A zoomed-in view (inset) shows how the columns at the surface are traced one-byone in sequence. $\mathbf{f}$ Surface profiles corresponding to the TEM images in $\mathbf{d}$ color coded by elapsed time. $\mathbf{g}$ Temporal evolution of the root mean square (RMS) roughness of the surface. $\mathbf{h}$ Temporal evolution of the bond angles $\beta_{j k}$ between the $j$ th column and its $k$ th nearest neighbor. Scale bars: $200 \mathrm{~nm}$.

the supracrystal-suspension interface are defined as the $(x, y)$ coordinates of the outermost layer of the supracrystal using a local order parameter of the solid bond number $\left(\xi_{j}\right.$ for the $j$ th column) to differentiate the columns in the supracrystal from those dispersed in the suspension. Solid bond number $\xi_{j}$ characterizes the number of the nearest-neighbors of the jth column that are connected to the $j$ th column in a crystalline local environment with similar bond orientations ${ }^{34,35}$. This order parameter serves as a rigorous measure of local crystallinity by eliminating false bond-order coherence originated from dense disordered phase ${ }^{36,37}$. Specifically, the connectivity $\left(S_{j k}\right)$ of a bond between the $j$ th column and its $k$ th nearest-neighbor ${ }^{35,38}$ is computed following $S_{j k}=\operatorname{Re}\left(\psi_{6 j} \psi_{6 k}^{*}\right)$ for a total of 441,704 column pairs in Supplementary Movie 2. The histogram of $S_{j k}$ shows a characteristic $S_{\mathrm{c}}$ of 0.175 , chosen as the local minimum between the two peaks of the histogram (Fig. 2a, b). Only the bonds of a high connectivity, i.e., $S_{j k}>S_{\mathrm{c}}$, are counted as a solid bond in calculating $\xi_{j}$ (Supplementary Fig. 8 and Supplementary Note 7). As shown in Fig. 2c, there exists a clear distinction in the values of $S_{j k}$ between the supracrystal and suspension phases. Figure $2 \mathrm{~d}$ shows the time-lapse liquid-phase TEM snapshots with the $j$ th column colored according to $\xi_{j}$. The columns of $\xi_{j} \geq 4$ are assigned quantitatively as the supracrystal phase. The instantaneous surface profiles are extracted from the coordinates of the columns at the outermost crystalline layer (black lines in Fig. 2e and Supplementary Note 7, Supplementary Movie 2), which are traced by starting from the leftmost column and connecting along the nearest neighboring bond of the smallest bond angle change (Fig. 2e, inset). 
Notably, the supracrystal undergoes growth and pushes the surface profile forward over time (Supplementary Fig. 3 and Supplementary Movie 2), yet the roughness of the surface stays constant for a long time, suggesting the fluctuation of the profile is driven by the generic balance between thermal agitation and surface energy. As shown in Fig. 2f, representative surface profiles are plotted with colors that indicate elapsed time. Further analysis of root mean square (RMS) roughness versus time shows a twostage evolution which we discuss more in detail later: RMS roughness stays constant at $(104 \pm 36) \mathrm{nm}$ during the first $\sim 100 \mathrm{~s}$ and stabilizes to a lower value of $(80 \pm 25) \mathrm{nm}$ for the later $\sim 50 \mathrm{~s}$ (Fig. 2g and Supplementary Fig. 9, Supplementary Note 8). This RMS roughness profile contrasts with the reported power law dependence of RMS roughness versus time when kinetic roughening by random arrival of material on the surface dominates the surface profile 27,39 . We attribute this observation to the time scale separation in the supracrystal growth and surface fluctuation (Supplementary Note 9). Similar time scale separation arguments have been used in systems of micron-sized colloids with ongoing crystal growth ${ }^{40}$ and with grain boundaries deformed under external shear ${ }^{30}$. The orientation of the supracrystal stays unchanged as shown in the bond network (Fig. 2h), facilitating our later analysis on the anisotropy of interfacial stiffness.

Shift of surface facets during supracrystal growth. For an interface involving a crystalline lattice, surface energy $\gamma$ depends on the direction of the interfacial plane relative to the crystal lattice and is anisotropic because of its structural origin. This anisotropy plays an important role in governing crystal nucleation and shaping crystals into distinctive morphologies ${ }^{8,41}$. Interfacial fluctuations thus depend on both $\gamma$ (related to interfacial stretching) and the second derivative of $\gamma$ with respect to surface orientation (related to interfacial bending), which are better characterized by interfacial stiffness $\tilde{\gamma}$ (sum of these two contributions ${ }^{42,43}$ ). Interestingly, in our system, although the lattice orientation of the supracrystal stays unchanged (Fig. 2h), its surface orientation undergoes a shift within our observation time to separate the supracrystal growth process into two stages, due to the very concept of anisotropic interfacial stiffness.

As shown in Fig. 3a, the temporal evolution of surface orientation $\theta$ exhibits two stages (labeled in salmon and aquamarine), each of which follows its own Gaussian distribution (Supplementary Fig. 9). Here $\theta$ is defined as the angle between the linear regression of the surface profile ${ }^{30}$ tracked in each TEM snapshot and a horizontal axis (Supplementary Note 10). The representative snapshots for these two stages are shown in Fig. 3b: The surface orientation $\theta$ undergoes a $19 \%$ increase, starting from $\theta=(36 \pm 6)^{\circ}$ for the first stage of $\sim 100 \mathrm{~s}$, and then shifting to stabilize at $\theta=(49 \pm 5)^{\circ}$ for the second stage of $\sim 50 \mathrm{~s}$ (Supplementary Fig. 9a). The surface orientation dictates the exposed facets (and the growth direction) of the supracrystal, which in our experiment changes from a high index (340) facet to a low index (210) facet, converging to thermodynamically favored lower surface energy state. The orientation shift is also consistent with the notations in atomic crystals that facets of lower Miller indexes tend to have a lower surface energy and be more favored to be exposed at the crystal surface $^{7}$. Despite the shift, the lattice constant $\sigma$ remains the same over the growth process (blue line in Fig. 3a), suggesting that the system is in a stable and nonchanging interaction environment without accumulated beam effects. It is noteworthy that the radiolysis products generated during liquid-phase TEM imaging reach at steady concentrations within seconds ${ }^{32,44}$. At our low dose rates, these products do not react with NPs but lead to an effective ionic strength increase that screens interprism electrostatic interaction, which triggers supracrystal growth ${ }^{31,32,45}$.
The surface of the supracrystal exhibits a lower roughness upon the shift of surface orientation. Typically, the surface roughness is determined by the surface energy existing between two phases against thermal fluctuations ${ }^{46}$. The surface profiles of $(x, y)$ coordinates depicted in Fig. $3 c$ are defined by both this balancing effect and the supracrystal growth, the latter of which we subtract to measure parameters generic to surface fluctuation (Supplementary Note 10). Specifically, we extract a modified surface profile as a one-dimensional (1D) height function $h(x, t)$ as detailed below. The directly mapped surface profiles are first rotated anti-clockwise by the averaged $\theta$ of each stage into $1 \mathrm{D}$ functions of $h^{\prime}(x, t)$ (Supplementary Fig. 10a). The increase of $h^{\prime}$ $(x, t)$ in the direction vertical to the surface is contributed solely by the crystal growth characterized by a constant velocity $v$ at each stage, following the literature for crystal growth from micron-sized colloids ${ }^{40}$. As shown in Supplementary Fig. 10b, the growth velocity is $5.30 \pm 0.23 \mathrm{~nm} \mathrm{~s}^{-1}$ at stage 1 and increases to $6.52 \pm 0.36 \mathrm{~nm} \mathrm{~s}^{-1}$ at stage 2 , indicating faster and more favored growth at the later stage. By subtracting the crystal growth contribution, the final obtained height function $h(x, t)$ fluctuates but does not monotonically increase or decrease (Supplementary Fig. 10c, Supplementary Note 10). The accumulated probability distributions of $h(x, t)$ for both two stages fit with a Gaussian distribution centered at $h(x, t)=0$ (Fig. $3 \mathrm{~d}$ ), suggesting that the crystal growth contribution is removed, and thermal fluctuations dictate the surface profile ${ }^{30}$. The RMS roughness in each of the two stages (Fig. $2 \mathrm{~h}$ and Supplementary Fig. $9 \mathrm{~b}$ ) does not exhibit monotonic changes over time, consistently validating no effects from crystal growth.

Both the instantaneous RMS roughness and the average roughness $R$ (full width at half maximum of the Gaussian fit for $h(x, t)$ ) show that the surface becomes smoother at stage 2 than at stage 1 (Supplementary Movie 4). Stage 1 has an average roughness $R_{1}$ of $305 \mathrm{~nm}$, about three times the lattice parameter of the supracrystal (Fig. 3d), while $R_{2}$ becomes $31 \%$ smaller in stage $2(210 \mathrm{~nm})$, elucidating that the supracrystal converges towards smooth surfaces during growth, consistent with flatfaceted polygonal shapes of equilibrated supracrystals formed outside TEM ${ }^{8,13,41,47}$ (e.g., cubic, octahedron, rhombic dodecahedron crystals formed by DNA-functionalized gold $\mathrm{NPs}^{41}$ ). Our in-situ TEM imaging sheds light on the dynamic process of the crystal surface development.

CWT and facet-dependent interfacial stiffness. We validate the applicability of CWT to our system, which allows us to extract quantitative measures of anisotropic interfacial stiffness and interfacial mobility. CWT has been successfully applied to simulation studies of atomic interfaces ${ }^{22}$ (e.g., $\mathrm{Ni}^{23,24}, \mathrm{Ag}^{25}, \mathrm{Fe}$ with different lattice structure ${ }^{26}$ ) and experimental studies of micron-sized colloidal suspensions ${ }^{20,21,40,42,48}$, but not for a nanoscale fluctuating interface at the single building block resolution. We conduct FFT to decompose $h(x, t)$, only accessible via our direct imaging, into a series of sinusoidal waves with different wave vectors as shown in Fig. 4a. From the equipartition theorem, the time-averaged Fourier coefficient $\langle A(k)\rangle$ for wave vector $k$ is expected to follow $\left\langle|A(k)|^{2}\right\rangle=\frac{k_{\mathrm{B}} T}{L \tilde{\gamma}} k^{-2}$, where $\tilde{\gamma}$ is the interfacial stiffness, $L$ is the length of the supracrystal surface (fixed at 1560 $\mathrm{nm}), k_{\mathrm{B}}$ is the Boltzmann constant, and $T$ is the temperature (Supplementary Note 11). Indeed, as we plot the measured \langle|$A$ $\left.\left.(k)\right|^{2}\right\rangle$ versus $k$ (Fig. $4 \mathrm{~b}$ ), we find that it follows a power law of -2 as expected from CWT, confirming both the roughness and the height function of the surface are determined by thermal equilibrium.

According to CWT, we calculate the interfacial stiffness $\tilde{\gamma}$ at the two growth stages, which elucidates an intriguing dependence on 
a
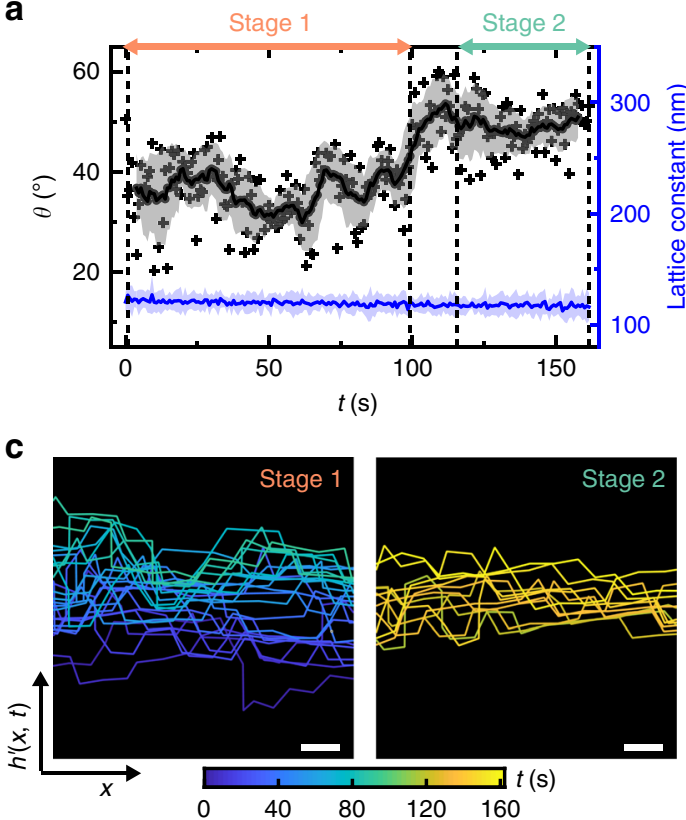

b
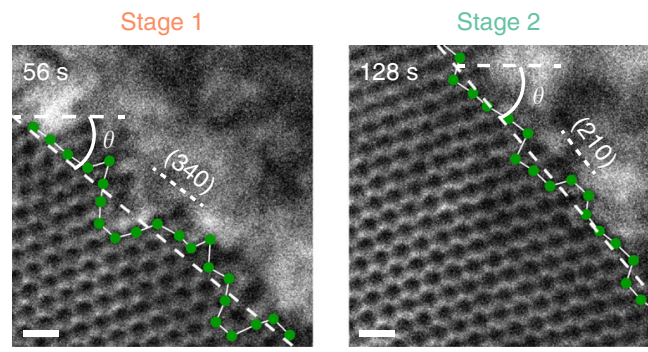

d

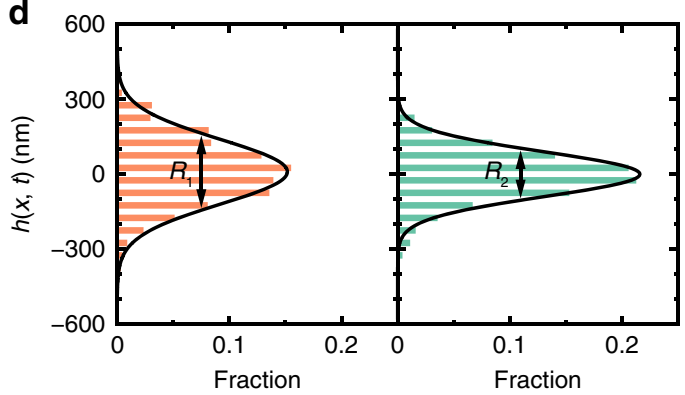

Fig. 3 Shift of surface orientation and facet during the supracrystal growth. a Plot showing the evolution of the surface orientation ( $\theta$, black crosses) over time $t$ as computed from the surface profiles. The black filled circles are the averaged $\theta$ over the successive $8.5 \mathrm{~s}$ with the gray shadow denoting s.d. Two stages of $\theta$ are observed and highlighted with salmon and aquamarine arrows. The lattice constant $\sigma$ in the supracrystal (blue line) shows no significant change during growth with the shadow denoting s.d. b Typical liquid-phase TEM images for stage 1 and stage 2 highlighting the surface orientation shift. The columns at the surface are labeled as green filled circles connected by white bonds. White dotted lines denote linear regression of the surface profiles. c Surface profiles of the supracrystal color coded by elapsed time ( $4 \mathrm{~s}$ interval between neighboring profiles). $\mathbf{d}$ Corresponding histogram of $h(x, t)$, with the black lines denoting the corresponding Gaussian distributions. The surface roughness $R_{1}$ for stage 1 and $R_{2}$ for stage 2 are labeled as the full width at halfmaximum. Scale bars: $200 \mathrm{~nm}$.
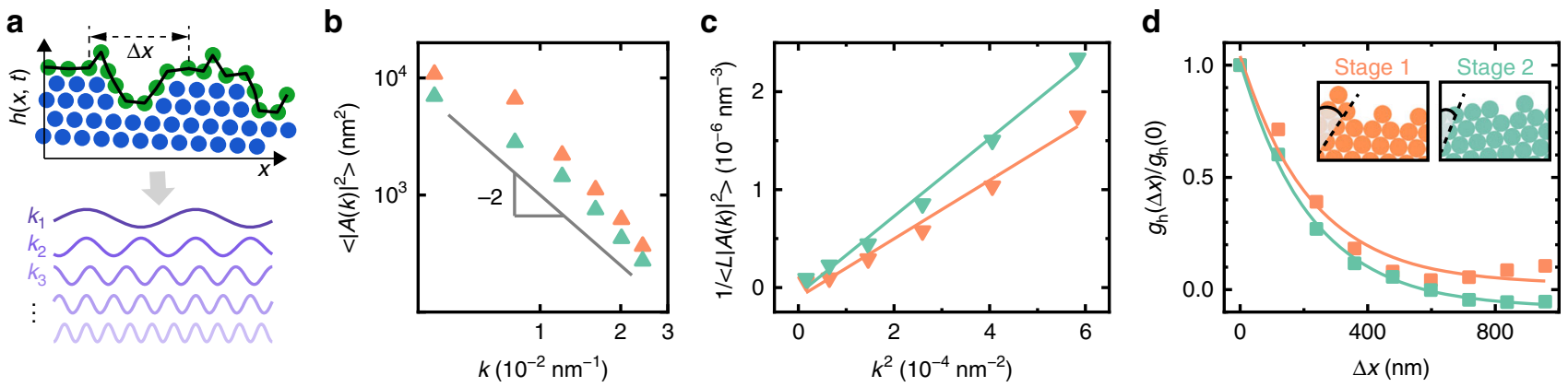

Fig. 4 Validation of capillary wave theory (CWT) at the nanoscale and calculation of orientation-dependent interfacial stiffness. a Schematic illustrating the Fourier decomposition of the height function $h(x, t)$ into superimposed waves with different wave vectors $\left(k_{1}, k_{2}, k_{3}, \ldots\right)$. b Dependence of $\left\langle|A(k)|^{2}\right\rangle$ on $k$ on a log-log scale plot for stage 1 (salmon) and stage 2 (aquamarine). The gray line of a power law of -2 is drawn to guide the eye. $\mathbf{c}$ Dependences of $1 / L\left\langle|A(k)|^{2}\right\rangle$ on $k^{2}$ overlaid with the corresponding linear fitting lines for the two stages. $\mathbf{d}$ Plot of lateral correlation functions $g_{\mathrm{h}}(\Delta x)$ normalized by $g_{\mathrm{h}}(0)$ as a function of $\Delta x$ (defined in $\mathbf{a}$ ) overlaid with exponential fitting for stage 1 (salmon) and stage 2 (aquamarine). Insets highlight the different surface orientations of two stages.

surface orientation. Specifically, we plot $1 / L\left\langle|A(k)|^{2}\right\rangle$ as a function of $k^{2}$, the linear fitting of which measures the interfacial stiffness $\tilde{\gamma} / k_{\mathrm{B}} T$ as the slope (Fig. 4c). On one hand, the interfacial stiffness values of both the first and second stages are on the order of $10^{-14} \mathrm{~J} \mathrm{~m}^{-1}$, consistent with interface stiffness scaling as $\sim k_{\mathrm{B}} T / l_{\mathrm{c}}$, where $l_{\mathrm{c}}$ is the size of the building block $(120 \mathrm{~nm}$ in our system). On the other hand, the interfacial stiffness increases from $(1.23 \pm 0.09) \times 10^{-14} \mathrm{~J} \mathrm{~m}^{-1}$ (stage 1) to $(1.63 \pm 0.08) \times 10$ ${ }^{-14} \mathrm{~J} \mathrm{~m}^{-1}$ (stage 2) by $32 \%$, leading to the lower surface roughness as noted above (Fig. 3d). Earlier work has shown that, for a given crystal interfaced with a suspension, the surface orientation with higher interfacial stiffness corresponds to that of lower surface energy ${ }^{42}$. This shift of surface orientation of the growing supracrystal thus effectively lowers the surface energy, matching with the Wulff construction rule to exhibit lowest surface energy facets for an equilibrium crystal shape.

It is noteworthy that molecular dynamics simulations have been mostly used to obtain the surface energy of a NP supracrystal to predict its equilibrium shape based on the various construction rules 8,49 . Our measurements of the orientationdependent energetic parameters directly from experiments can aid engineering of the surfaces and final morphology of NP supracrystals as well as understanding nanoscale interactions, where discreteness and nonadditivity have made modeling 
challenging ${ }^{16,50}$. As an example, our CWT-based analysis can reveal a correlation length $\zeta$ along the supracrystal surface, which measures the characteristic exponential decay length of the lateral static correlation $g_{\mathrm{h}}(\Delta x)=\langle h(x, t) h(x+\Delta x, t)\rangle_{x, t}$. As shown in Fig. $4 \mathrm{~d}$, both the data from the first and second stages follow an exponential decay, which agree with the prediction of CWT, exhibiting similar correlation lengths $(225 \pm 40 \mathrm{~nm}$ for stage 1 ; $224 \pm 13 \mathrm{~nm}$ for stage 2). These correlation lengths are about two building block size, suggesting a short-ranged intercolumn interaction consistent with our previous results, where the attraction damps to $10 \%$ when the distance of the columns is at $25 \%$ of column size ${ }^{31}$. We also measure the parameter of interfacial mobility relating the interfacial normal velocity to the driving force (Supplementary Fig. 11 and Supplementary Note 12), which is $(0.30 \pm 0.16) \mathrm{m}^{3} \mathrm{~J}^{-1} \mathrm{~s}^{-1}$ for stage 1 , consistent with a scaling analysis, suggesting there exists no bulk driving forces other than thermal fluctuation, such as elastic strains, stored energy of deformation, electromagnetic fields or thermochemical gradients ${ }^{51}$.

\section{Discussion}

Using low-dose liquid-phase TEM imaging, single-particle tracking, and quantitative structural analysis, we are able to identify and monitor real-space surface profiles at the nanoscale. Thermal capillary waves are observed with single particle resolution for a NP supracrystal, which allows experimental measurements of a series of otherwise inaccessible physical parameters determining the surface and shape of supracrystals. Impressively, the anisotropic interfacial stiffness we quantify in the experiments shows the growth of supracrystal towards exhibiting flatter surfaces with lower surface energy, consistent with the Wulff construction rule ${ }^{12,50,52}$. Beyond the quasi-equilibrium system we focus on here, where the surface profile is controlled by the balance between surface energy and thermal fluctuations, the imaging of surface profiles can allow mapping of other parameters on other fluctuating systems at the nanoscale. For example, one can measure the bending and stretching modulus in a fluctuating vesicle where inter-lipid interactions add rigidity to the vesicle during vesicle transformation ${ }^{29}$, or measure the deposition laws as the RMS roughness changes with time due to active materials deposition ${ }^{27,53}$. More studies can emerge to use our method to study fluctuations as liquid-phase TEM becomes more compatible with biological samples and out-of-equilibrium field application.

Our capability to measure facet-dependent interfacial stiffness and our demonstration on their role in shaping the supracrystals can advance crystal design at the nanoscale. For example, one can control the interfacial stiffness and crystal habit by utilizing the toolkits of both intrinsic parameters of NP shape and surface chemistry ${ }^{41,54-56}$ as well as extrinsic parameters, such as temperature, $\mathrm{pH}$, and ionic strength ${ }^{57-59}$. Previously reports have shown that changing the length of DNA ligands on the same gold NPs has led to tunability in the lattice symmetry of the interior structure and exposed facets of the supracrystal ${ }^{17,55,60}$. In binary systems, a diversity of supracrystal shapes including the exotic diamond-like lattices has been achieved by introducing two ligand types, two differently sized NPs, or two NP compositions ${ }^{61}$. Our approach can elucidate the fundamental parameter of interfacial stiffness in these systems and make the crystal design predictable. Broadly, complete 2D surface profiles for more complicated supracrystal structures can be possibly achieved by TEM advancements such as SINGLE ${ }^{62}$, ultrafast electron tomography ${ }^{63}$ and focus-based 3D imaging with $z$-slices $^{64}$, which can be analyzed following the protocols presented here. In-situ liquid-phase TEM is also compatible with introducing environmental triggers during the observation ${ }^{18,65}$, to dynamically modify the surface roughness and interfacial stiffness of crystals as one sees the growth.

\section{Methods}

Chemicals. All chemicals were purchased and used without further purification. Sodium iodide $(99.999 \%$, NaI), cetyltrimethylammonium bromide (BioXtra, $\geq 99 \%$, $\mathrm{CTAB})$, gold (III) chloride trihydrate $\left(\geq 99.9 \%, \mathrm{HAuCl}_{4}\right)$, sodium citrate tribasic dihydrate (BioUltra, $\geq 99.5 \%$ ), sodium borohydride $\left(99 \%, \mathrm{NaBH}_{4}\right)$, L-ascorbic acid (BioXtra, $\geq 99.0 \%$ ) and sodium hydroxide $(99.99 \%, \mathrm{NaOH})$ were purchased from Sigma Aldrich. Sodium chloride (99.3\%, NaCl, Fisher Scientific), sodium phosphate monobasic monohydrate $\left(99.0-102.0 \%, \mathrm{NaH}_{2} \mathrm{PO}_{4} \cdot \mathrm{H}_{2} \mathrm{O}\right.$, EMD Millipore), sodium phosphate dibasic anhydrous ( $>99 \%, \mathrm{Na}_{2} \mathrm{HPO}_{4}$, Acros), and 2-(2-[2-(11-mercaptoundecyloxy)-ethoxy]-ethoxy)-ethoxy-ethoxy-ethoxy-ethoxy-acetic acid ( $\geq 95 \%$, HS $\left(\mathrm{CH}_{2}\right)_{11}\left(\mathrm{OC}_{2} \mathrm{H}_{4}\right)_{6} \mathrm{OCH}_{2} \mathrm{COOH}$, Prochimia Surfaces) were purchased and used without further purification. The water used in this work is nanopure water (18.2 $\mathrm{M} \Omega \mathrm{cm}$ at $25^{\circ} \mathrm{C}$ ) purified by a Milli-Q Advantage A10 system. All glassware used in this work was treated with aqua regia (mixture of $\mathrm{HCl}$ and $\mathrm{HNO}_{3}$ with a volume ratio of $3: 1$ ), fully rinsed with water and dried.

Synthesis and purification of gold triangular nanoprisms. The gold triangular nanoprisms used in our experiments were synthesized and purified via a seeded growth method according to the literatures ${ }^{32,45,66-68}$. First, a gold NP seed solution was prepared by rapidly mixing an aqueous solution of $\mathrm{HAuCl}_{4}(250 \mu \mathrm{L}, 10 \mathrm{mM})$, sodium citrate $(500 \mu \mathrm{L}, 10 \mathrm{mM})$ and ice cold $\mathrm{NaBH}_{4}(300 \mu \mathrm{L}, 10 \mathrm{mM})$ sequentially with $18.95 \mathrm{~mL}$ water in a $50 \mathrm{~mL}$ flask and stirred at $1150 \mathrm{rpm}$ for $1 \mathrm{~min}$. The addition of the $\mathrm{NaBH}_{4}$ solution should be fast to obtain small and monodisperse gold seeds. The seed solution was incubated at $40-45^{\circ} \mathrm{C}$ for $15 \mathrm{~min}$ and cooled down to room temperature. Gold triangular nanoprisms were grown from the gold seeds (usually good within $2 \mathrm{~h}$ after preparation). Aqueous solutions of $\mathrm{HAuCl}_{4}$ $(250 \mu \mathrm{L}, 10 \mathrm{mM}), \mathrm{NaOH}(50 \mu \mathrm{L}, 100 \mathrm{mM})$, ascorbic acid $(50 \mu \mathrm{L}, 100 \mathrm{mM})$, and 22 $\mu \mathrm{L}$ of the as-synthesized gold seed solution were sequentially added into $9 \mathrm{~mL}$ of 50 $\mathrm{mM}$ CTAB solution containing $50 \mu \mathrm{M} \mathrm{NaI}$ in a $20 \mathrm{~mL}$ scintillation vial. The solution was hand-shaken for $1 \mathrm{~s}$ after each addition and the mixture was left undisturbed for $30 \mathrm{~min}$. The color of the solution gradually changed from colorless to purple, indicating the formation of triangular nanoprisms along with spherical impurities. To purify the nanoprisms, the purple solution was transferred to a 15 $\mathrm{mL}$ centrifuge tube and $0.9 \mathrm{~mL}$ of $2 \mathrm{M} \mathrm{NaCl}$ was added. After the solution was wellmixed, it was left undisturbed for $2 \mathrm{~h}$ to induce face-to-face stacking of triangular nanoprisms due to screening of electrostatic repulsion ${ }^{32}$. This solution was centrifuged twice (1st round: $4900 \mathrm{rpm}$ for $30 \mathrm{~s}$; 2nd round: $1350 \mathrm{rpm}$ for $5 \mathrm{~s}$ ). Immediately after each centrifugation, the supernatant was removed as much as possible using a micropipette. After the $2^{\text {nd }}$ round of centrifugation, several drops of water were first added to the sediments to redisperse the product and $9 \mathrm{~mL}$ of 50 $\mathrm{mM}$ CTAB was added to keep the prisms stable for long-time storage.

Surface modification of the gold triangular nanoprisms. Ligand exchange was conducted for the nanoprisms with carboxylate-terminated thiols following a literature $\operatorname{method}^{32,45,69}$. The nanoprisms were stored in in $50 \mathrm{mM}$ CTAB solution after synthesis as detailed above. Firstly, two centrifugations were conducted to decrease the concentration of CTAB molecules dissolved in the solution (1st round: $8800 \mathrm{rpm}$ for $8 \mathrm{~min}$; 2 nd round: $6600 \mathrm{rpm}$ for $8 \mathrm{~min}$ ). After the 1 st round of centrifugation, the supernatant was removed and the remaining liquid with sediments $(\sim 50 \mu \mathrm{L})$ was mixed with $8.95 \mathrm{~mL}$ of water. After the $2^{\text {nd }}$ round of centrifugation, the supernatant was removed and the remaining liquid with sediments $(\sim 50 \mu \mathrm{L})$ was mixed with $3.00 \mathrm{~mL}$ of water. Secondly, an aqueous solution of thiol molecules $(44.26 \mu \mathrm{L}, 7.93 \mathrm{mM})$ was added to the prism solution and incubated for $30 \mathrm{~min}$. Finally, the solution was sonicated for $5 \mathrm{~s}$ and $0.538 \mathrm{~mL}$ of $1 \mathrm{M} \mathrm{pH}=8$ phosphate buffer solution (PBS, composed of $0.07 \mathrm{M} \mathrm{NaH} \mathrm{PO}_{4} \cdot \mathrm{H}_{2} \mathrm{O}$ and $0.93 \mathrm{M}$ $\mathrm{Na}_{2} \mathrm{HPO}_{4}$ ) was gently added to the solution and left undisturbed overnight. The final solution contained $100 \mu \mathrm{M}$ of thiol molecules and $0.15 \mathrm{M}$ of $\mathrm{pH}=8 \mathrm{PBS}$, where the PBS solution screens the electrostatic repulsion of deprotonated thiol ligands and to facilitate efficient coating of the gold prism surface. During $\sim 15 \mathrm{~h}$ of incubation, the prisms not only were fully covered by thiols but also began to assemble face-to-face stacked and formed into black sediments. Just prior to use for liquid-phase TEM, we diluted this solution to a PBS concentration of $34.5 \mathrm{mM}$, so that the prisms remained dispersed instead of face-to-face stacked.

Liquid-phase TEM sample preparation and imaging. Liquid-phase TEM imaging was carried out on a JEOL 2100 Cryo TEM with a spot size 3 with a $\mathrm{LaB}_{6}$ emitter at $200 \mathrm{kV}$ using the Protochips Poseidon 210 liquid flow holder. The illumination area is intentionally kept larger than the fluorescent screen of the TEM $(\sim 35 \mathrm{~cm})$. The electron beam is sufficiently spread out to minimize artefacts from the boundary and nonuniformity of the illumination area on the supracrystal growth and to achieve sufficiently low dose rates. The movies were captured by a Gatan Ultrascan charge-coupled device (CCD) camera with a $0.1 \mathrm{~s}$ exposure time per frame at a rate of 1.3 frames per seconds (fps). In a typical experiment, an aliquot of the nanoprism solution prepared above ( $34.5 \mathrm{mM} \mathrm{pH}=8$ PBS buffer solution) 
was micropipetted on a $\mathrm{SiN}_{x}$ chip (window: $550 \mu \mathrm{m} \times 20 \mu \mathrm{m}, 150 \mathrm{~nm}$ spacer flow echip, Protochips), which was then assembled with another $\operatorname{SiN}_{x}$ chip (window: $550 \mu \mathrm{m} \times 20 \mu \mathrm{m}$ ) in a Protochips Poseidon 210 liquid flow TEM holder. The $\mathrm{SiN}_{x}$ chips were pretreated at a medium RF level for $45 \mathrm{~s}$ using a Harrick PDC-23G basic plasma cleaner to render them clean and hydrophilic. During the liquid-phase TEM imaging, the electron dose rates were kept low $\left(3.7-14.8 \mathrm{e}^{-} \AA^{-2} \mathrm{~s}^{-1}\right)$. At this dose rate, the thiol ligands on the nanoprism have been shown to stay intact on the particle surface and remain negatively charged in our previous studies $31,32,45$.

\section{Data availability}

The data that support the findings of this study are available from the corresponding authors upon request.

\section{Code availability}

Customized Matlab source codes for image processing and particle tracking are freely available at https://github.com/chenlabUIUC/particle-identification, including detailed instruction.

Received: 2 December 2019; Accepted: 7 August 2020;

Published online: 11 September 2020

\section{References}

1. Ross, M. B., Ku, J. C., Vaccarezza, V. M., Schatz, G. C. \& Mirkin, C. A. Nanoscale form dictates mesoscale function in plasmonic DNA-nanoparticle superlattices. Nat. Nanotech. 10, 453-458 (2015).

2. Park, D. J. et al. Plasmonic photonic crystals realized through DNAprogrammable assembly. Proc. Natl Acad. Sci. USA 112, 977-981 (2015).

3. Lim, J., Hippalgaonkar, K., Andrews, S. C., Majumdar, A. \& Yang, P. Quantifying surface roughness effects on phonon transport in silicon nanowires. Nano Lett. 12, 2475-2482 (2012).

4. Singh, A. V. et al. Bottom-up engineering of the surface roughness of nanostructured cubic zirconia to control cell adhesion. Nanotech. 23, 475101 (2012).

5. Pileni, M. P. Self organization of inorganic nanocrystals: Unexpected chemical and physical properties. J. Colloid Interface Sci. 388, 1-8 (2012).

6. Givargizov, E. I., Grinberg, S. \& Wester, D. W. Growth of Crystals (Springer, 2002).

7. Barmparis, G. D., Lodziana, Z., Lopez, N. \& Remediakis, I. N. Nanoparticle shapes by using Wulff constructions and first-principles calculations. Beilstein J. Nanotechnol. 6, 361-368 (2015).

8. Auyeung, E. et al. DNA-mediated nanoparticle crystallization into Wulff polyhedra. Nature 505, 73-77 (2013).

9. Marks, L. D. \& Peng, L. Nanoparticle shape, thermodynamics and kinetics. J. Phys.: Condens. Matter 28, 053001 (2016).

10. Prieto, J. E. \& Markov, I. Stranski-Krastanov mechanism of growth and the effect of misfit sign on quantum dots nucleation. Surf. Sci. 664, 172-184 (2017).

11. Heo, H. et al. Frank-van der Merwe growth versus Volmer-Weber growth in successive stacking of a few-layer $\mathrm{Bi}_{2} \mathrm{Te}_{3} / \mathrm{Sb}_{2} \mathrm{Te}_{3}$ by van der Waals heteroepitaxy: the critical roles of finite lattice-mismatch with seed substrates. Adv. Electron. Mater. 3, 1600375 (2017).

12. Boles, M. A., Engel, M. \& Talapin, D. V. Self-assembly of colloidal nanocrystals: From intricate structures to functional materials. Chem. Rev. 116, 11220-11289 (2016).

13. Haubold, D. et al. The formation and morphology of nanoparticle supracrystals. Adv. Func. Mater. 26, 4890-4895 (2016).

14. Wang, M. X. et al. Epitaxy: Programmable atom equivalents versus atoms. ACS Nano 11, 180-185 (2017).

15. $\mathrm{Wu}, \mathrm{L}$. et al. High-temperature crystallization of nanocrystals into threedimensional superlattices. Nature 548, 197-201 (2017).

16. French, R. H. et al. Long range interactions in nanoscale science. Rev. Mod. Phys. 82, 1887-1944 (2010).

17. Senesi, A. J. et al. Stepwise evolution of DNA-programmable nanoparticle superlattices. Angew. Chem. Int. Ed. 52, 6624-6628 (2013).

18. Ross, F. M. Opportunities and challenges in liquid cell electron microscopy. Science 350, aaa9886 (2015).

19. De Yoreo, J. J. \& N. A. J. M, S. Investigating materials formation with liquidphase and cryogenic TEM. Nat. Rev. Mater. 1, 16035 (2016).

20. Hernandez-Guzman, J. \& Weeks, E. R. The equilibrium intrinsic crystal-liquid interface of colloids. Proc. Natl Acad. Sci. USA 106, 15198-15202 (2009).

21. Hilou, E., Du, D., Kuei, S. \& Biswal, S. L. Interfacial energetics of twodimensional colloidal clusters generated with a tunable anharmonic interaction potential. Phys. Rev. Mater. 2, 025602 (2018).
22. Hoyt, J. J., Trautt, Z. T. \& Upmanyu, M. Fluctuations in molecular dynamics simulations. Math. Comput. Simul. 80, 1382-1392 (2010).

23. Hoyt, J. J., Asta, M. \& Karma, A. Method for computing the anisotropy of the solid-liquid interfacial free energy. Phys. Rev. Lett. 86, 5530 (2001).

24. Zykova-Timan, T., Rozas, R. E., Horbach, J. \& Binder, K. Computer simulation studies of finite-size broadening of solid-liquid interfaces: From hard spheres to nickel. J. Phys.: Condens. Matter 21, 464102 (2009).

25. Hoyt, J. J. \& Asta, M. Atomistic computation of liquid diffusivity, solid-liquid interfacial free energy, and kinetic coefficient in Au and Ag. Phys. Rev. B 65 , 214106 (2002)

26. Sun, D. Y., Asta, M., Hoyt, J. J., Mendelev, M. I. \& Srolovitz, D. J. Crystal-melt interfacial free energies in metals: fcc versus bcc. Phys. Rev. B 69, 020102 (2004).

27. Schneider, N. M. et al. Nanoscale evolution of interface morphology during electrodeposition. Nat. Commun. 8, 2174 (2017)

28. Ferrara, K. W., Borden, M. A. \& Zhang, H. Lipid-shelled vehicles: Engineering for ultrasound molecular imaging and drug delivery. Acc. Chem. Res. 42, 881-892 (2009).

29. Monzel, C. \& Sengupta, K. Measuring shape fluctuations in biological membranes. J. Phys. D. 49, 243002 (2016).

30. Gokhale, S., Nagamanasa, K. H., Santhosh, V., Sood, A. K. \& Ganapathy, R. Directional grain growth from anisotropic kinetic roughening of grain boundaries in sheared colloidal crystals. Proc. Natl Acad. Sci. U. S. A. 109, 20314-20319 (2012)

31. Ou, Z., Wang, Z., Luo, B., Luijten, E. \& Chen, Q. Kinetic pathways of crystallization at the nanoscale. Nat. Mater. 19, 450-455 (2020).

32. Kim, J., Jones, M. R., Ou, Z. \& Chen, Q. In situ electron microscopy imaging and quantitative structural modulation of nanoparticle superlattices. ACS Nano 10, 9801-9808 (2016).

33. Savage, J. R., Blair, D. W., Levine, A. J., Guyer, R. A. \& Dinsmore, A. D. Imaging the sublimation dynamics of colloidal crystallites. Science 314, 795-798 (2006)

34. Tan, P., Xu, N. \& Xu, L. Visualizing kinetic pathways of homogeneous nucleation in colloidal crystallization. Nat. Phys. 10, 73-79 (2013).

35. Lechner, W. \& Dellago, C. Accurate determination of crystal structures based on averaged local bond order parameters. J. Chem. Phys. 129, 114707 (2008).

36. ten Wolde, P. R., Ruiz-Montero, M. J. \& Frenkel, D. Simulation of homogeneous crystal nucleation close to coexistence. Faraday Discuss. 104, 93-110 (1996).

37. ten Wolde, P. R., Ruiz-Montero, M. J. \& Frenkel, D. Numerical calculation of the rate of crystal nucleation in a Lennard-Jones system at moderate undercooling. J. Chem. Phys. 104, 9932-9947 (1996).

38. Tang, X. et al. Optimal feedback controlled assembly of perfect crystals. ACS Nano 10, 6791-6798 (2016).

39. Family, F. Dynamic scaling and phase transitions in interface growth. Phys. A 168, 561-580 (1990).

40. Nguyen, V. D., Hu, Z. B. \& Schall, P. Single crystal growth and anisotropic crystal-fluid interfacial free energy in soft colloidal systems. Phys. Rev. E $\mathbf{8 4}$ 011607 (2011)

41. O’Brien, M. N., Lin, H.-X., Girard, M., Olvera de la Cruz, M. \& Mirkin, C. A Programming colloidal crystal habit with anisotropic nanoparticle building blocks and DNA bonds. J. Am. Chem. Soc. 138, 14562-14565 (2016).

42. Nguyen, V. D., Dang, M. T., Weber, B., Hu, Z. \& Schall, P. Visualizing the structural solid-liquid transition at colloidal crystal/fluid interfaces. Adv. Mater. 23, 2716-2720 (2011).

43. Liao, M., Xiao, X., Chui, S. T. \& Han, Y. Grain-boundary roughening in colloidal crystals. Phys. Rev. X 8, 021045 (2018).

44. Schneider, N. M. et al. Electron-water interactions and implications for liquid cell electron microscopy. J. Phys. Chem. C. 118, 22373-22382 (2014).

45. Kim, J., Ou, Z., Jones, M. R., Song, X. \& Chen, Q. Imaging the polymerization of multivalent nanoparticles in solution. Nat. Commun. 8, 761 (2017).

46. Aarts, D. G. A. L., Schmidt, M. \& Lekkerkerker, H. N. W. Direct visual observation of thermal capillary waves. Science 304, 847-850 (2004).

47. Seo, S. E., Girard, M., Olvera de la Cruz, M. \& Mirkin, C. A. Non-equilibrium anisotropic colloidal single crystal growth with DNA. Nat. Commun. 9, 4558 (2018).

48. Ramsteiner, I. B., Weitz, D. A. \& Spaepen, F. Stiffness of the crystal-liquid interface in a hard-sphere colloidal system measured from capillary fluctuations. Phys. Rev. E 82, 041603 (2010).

49. Li, T. \& Olvera de la Cruz, M. Surface energy fluctuation effects in single crystals of DNA-functionalized nanoparticles. J. Chem. Phys. 143, 243156 (2015)

50. Batista, C. A., Larson, R. G. \& Kotov, N. A. Nonadditivity of nanoparticle interactions. Science 350, 1242477 (2015).

51. Trautt, Z. T., Upmanyu, M. \& Karma, A. Interface mobility from interface random walk. Science 314, 632-635 (2006).

52. Nagaoka, Y. et al. Superstructures generated from truncated tetrahedral quantum dots. Nature 561, 378-382 (2018).

53. Efraim, Y. \& Taitelbaum, H. Persistence in reactive-wetting interfaces. Phys Rev. E 84, 050602 (2011) 
54. Shevchenko, E. V., Talapin, D. V., Kotov, N. A., O’Brien, S. \& Murray, C. B. Structural diversity in binary nanoparticle superlattices. Nature 439, 55-59 (2006).

55. O'Brien, M. N. et al. Exploring the zone of anisotropy and broken symmetries in DNA-mediated nanoparticle crystallization. Proc. Natl Acad. Sci. U. S. A. 113, 10485-10490 (2016).

56. Lu, F. et al. Unusual packing of soft-shelled nanocubes. Sci. Adv. 5, eaaw2399 (2019).

57. Young, K. L. et al. Assembly of reconfigurable one-dimensional colloidal superlattices due to a synergy of fundamental nanoscale forces. Proc. Natl Acad. Sci. U. S. A. 109, 2240-2245 (2012).

58. Chen, Y. \& Mao, C. pH-induced reversible expansion/contraction of gold nanoparticle aggregates. Small 4, 2191-2194 (2008).

59. Seo, S. E., Girard, M., de la Cruz, M. O. \& Mirkin, C. A. The importance of salt-enhanced electrostatic repulsion in colloidal crystal engineering with DNA. ACS Cent. Sci. 5, 186-191 (2019).

60. Macfarlane, R. J. et al. Nanoparticle superlattice engineering with DNA. Science 334, 204-208 (2011).

61. Kalsin, A. M. et al. Electrostatic self-assembly of binary nanoparticle crystals with a diamond-like lattice. Science 312, 420-424 (2006).

62. Park, J. et al. 3D structure of individual nanocrystals in solution by electron microscopy. Science 349, 290-295 (2015).

63. Migunov, V. et al. Rapid low dose electron tomography using a direct electron detection camera. Sci. Rep. 5, 14516 (2015).

64. Zaluzec, N. J. Scanning confocal electron microscopy. Microsc. Microanal. 13, 1560-1561 (2007).

65. Ou, Z. et al. Reconfigurable nanoscale soft materials. Curr. Opin. Solid State Mater. Sci. 23, 41-49 (2019).

66. Jones, M. R. \& Mirkin, C. A. Bypassing the limitations of classical chemical purification with DNA-programmable nanoparticle recrystallization. Angew. Chem. Int. Ed. 52, 2886-2891 (2013).

67. O’Brien, M. N., Jones, M. R., Kohlstedt, K. L., Schatz, G. C. \& Mirkin, C. A. Uniform circular disks with synthetically tailorable diameters: Twodimensional nanoparticles for plasmonics. Nano Lett. 15, 1012-1017 (2015).

68. Millstone, J. E. et al. Observation of a quadrupole plasmon mode for a colloidal solution of gold nanoprisms. J. Am. Chem. Soc. 127, 5312-5313 (2005).

69. Jones, M. R., Macfarlane, R. J., Prigodich, A. E., Patel, P. C. \& Mirkin, C. A. Nanoparticle shape anisotropy dictates the collective behavior of surfacebound ligands. J. Am. Chem. Soc. 133, 18865-18869 (2011).

\section{Acknowledgements}

This work was supported by the U.S. Department of Energy, Office of Basic Energy Sciences, Division of Materials Sciences and Engineering, under Award DE-FG02
07ER46471, through the Materials Research Laboratory at the University of Illinois. Experiments were carried out in part in the Materials Research Laboratory Central Research Facilities, University of Illinois.

\section{Author contributions}

Z.O. and Q.C. designed and performed the experiments. Z.O., L.Y., H.A., and B. S performed particle tracking and analysis of experimental data. All contributed to the writing of the paper.

\section{Competing interests}

The authors declare no competing interests.

\section{Additional information}

Supplementary information is available for this paper at https://doi.org/10.1038/s41467 020-18363-2.

Correspondence and requests for materials should be addressed to Q.C.

Peer review information Nature Communications thanks Sibani Biswal and other, anonymous, reviewer(s) for their contributions to the peer review of this work. Peer review reports are available.

Reprints and permission information is available at http://www.nature.com/reprints

Publisher's note Springer Nature remains neutral with regard to jurisdictional claims in published maps and institutional affiliations.

(c) Open Access This article is licensed under a Creative Commons Attribution 4.0 International License, which permits use, sharing, adaptation, distribution and reproduction in any medium or format, as long as you give appropriate credit to the original author(s) and the source, provide a link to the Creative Commons license, and indicate if changes were made. The images or other third party material in this article are included in the article's Creative Commons license, unless indicated otherwise in a credit line to the material. If material is not included in the article's Creative Commons license and your intended use is not permitted by statutory regulation or exceeds the permitted use, you will need to obtain permission directly from the copyright holder. To view a copy of this license, visit http://creativecommons.org/ licenses/by/4.0/.

(C) The Author(s) 2020 